\title{
Energy Management Services based on Home Energy Grid Technology
}

\author{
Hyunjeong Lee *, Wan-Ki Park *, Il-Woo Lee* \\ *Energy IT Research Section, Smart Green Life Research Department, \\ IT Convergence Technology Research Laboratory, ETRI \\ 218 Gajeongno, Yuseong-gu, Daejeon, 305-700 KOREA
}

\{hjlee294, wkpark, ilwoo\}@etri.re.kr

\begin{abstract}
In this paper, smart energy management services are illustrated based on home energy grid technology. Smart plugs are used to get the data of the appliances' power consumption. First, smart plugs are installed for each appliance in the smart home, detect the data of power consumption, and send them to the home energy grid server. And, the server processes the data to monitor the energy consumption and report the residents. Also, the server provides the interface to control appliances. Using the scheme, users can monitor their energy consumption, and control their appliances to avoid energy leakage.
\end{abstract}

Keywords — Smart, Energy Saving, Smart Grid, Home, Smart Plug

\section{INTRODUCTION}

Recently, smart and green technologies are the main research issues to save energy and reduce carbon emission. Therefore, many studies for these technologies are being carried out, for example, smart grid, energy saving, and zero carbon technologies [1]. Residence and commercial buildings are also the main areas for energy saving, because they consume over $22 \%$ of national energy [2]. To realize these technologies, monitoring and statistics of energy consumption is the basis for reducing energy leakage and planning energy utilization.

In this paper, smart energy management services are illustrated based on home energy grid (HEG) technology. Smart plug (SP) is the new product for these purposes. Users can remotely monitor and control their appliances using their mobile devices and personal computers. First, a SP is installed for each appliance, connects and sends the data of power consumption to the HEG server. Then, the server processes, saves, and manages the data. Using the method, users can monitor and control the appliances through the server and SPs. Also, energy costs can be saved by monitoring and turning off the appliances when not in use.

The structure of this paper is organized as follows. In Section 2, smart and green technologies are described as related works. Scenarios and issues for HEG services are illustrated in Section 3. System architecture and a sequence flow for this purpose are described in Section 4. At last, conclusions will be presented in Section 5.

\section{RELATED WORKS}

\section{A. Smart Grid}

To provide high quality and reliable electrical energy service, the information and technology era has been a rise [3]. Also, the demand for preserving natural resources and the environment has been increased. In accordance with these trends, the smart grid systems are developed that include a physical power system and an information system that link a variety of equipment and means together to form a customer service platform. Smart grid is a technology to obtain various beneficial effects by giving smart to the existing power grid [4]. One of the goals for energy efficiency of many companies and nations is the creation of a smart energy distribution grid [5]. Smart grid technology can provide new functions such as self-healing, high reliability, energy management, and real-time pricing. Many technologies related to power and energy topics are converged for smart grid such as electrical engineering, information technology, communication, control and automation. The main contributions of the smart grid are to reduce $\mathrm{CO} 2$ emission and save energy, which are beneficial to the government in that costs of imported raw materials used in the production of energy, to the energy suppliers to minimize the reserved power, and to the customers to save energy costs by monitoring the real-time energy use, controlling the appliances, and managing the scheduling pattern of their home electricity usage, based on the real-time prices (RTPs).

\section{B. Smart Energy Profile 2.0}

The smart grid helps consumers to manage their usage and generation of energy and it is a basis of innovation for new products and services in energy management [6]. To enable this capability, information flow between devices such as meters, smart appliances, plug-in electric vehicles, energy management systems, and distributed energy resources, including renewable energy and storage elements, have to occur in an open, standardized, secure, and interoperable method.

Smart energy profile (SEP) 2.0 is an application-layer profile that includes a collection of standards where options 
and configurations are specified [7]. And, it focuses on communications related to efficiency, usage, price, demand response, and load control (DRLC), messaging, and so on. Also, it is independent of MAC/PHY layer, runs over generic Internet Protocol stack, and provides standardized definitions of devices and interfaces to communications among devices. The SEP is designed to implement a representational state transfer (REST) architecture, which is built around the core actions of GET, HEAD, PUT, POST, and DELETE, with the addition of a lightweight subscription mechanism. Any application protocol that can implement a RESTful command set could likely be used with SEP 2.0, but HTTP is a required baseline for interoperable SEP 2.0 implementations. HTTP utilizes TCP as its transport protocol. As a result, TCP manages the session providing delivery assurance and windowing.

In this paper, SEP 2.0 is used to describe appliances and communications for energy services, such as DRLC and RTP.

\section{SERVICE SCENARIOS FOR THE HOME ENERGY GRID}

HEG system helps residents to save energy by providing the information of energy consumption, interfaces to control home appliances, and automatic control services when not in use. Figure 1 shows the configuration of the devices for HEG services, such as appliances, a HEG server, SPs, sensors, a router and energy service providers (ESPs). HEG server has various kinds of wireless, such as Wi-Fi, ZigBee, smart utility network (SUN), and infrared (IR), processes energy related data, and provides residents energy management services. SPs are installed per one device, collect meter data, and control the power supply to appliances. Two types of sensors are used to collect data of occupancy and temperature, and send the data to the HEG server for using in energy saving services. And, a router provides Wi-Fi connections among HEG server, mobile devices, and smart appliances. Finally, ESPs provide users energy saving and management services, such as DRLC and RTP.

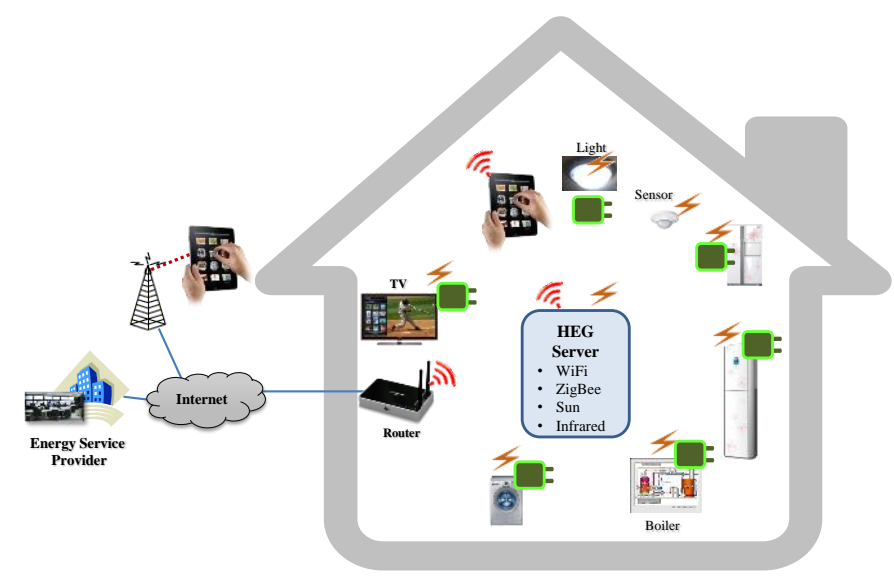

Figure 1. Configuration of the devices for HEG services

Many energy saving scenarios can be provided using the HEG services. A resident, Sally, wants to know the current electricity usage of her home. She connects to the HEG server using her mobile device, and monitors the information. She also can know the energy usage for each appliance, and turn off the appliance when not in use. She also can schedule for controlling the appliances corresponding to DR and RTP services by the ESPs. When there is none in the bedroom, the occupancy sensors detect and send the information. Then, the HEG server recognizes the absence situation, searches the appliances, that are predefined to be turned off in the absence context, and notifies to Sally that the appliances will be turned off. She positively responds the message, and the server performs the action. Using this mechanism, energy conservation and management can be carried out for the home.

\section{IV.DESIGN AND IMPLEMENTATION OF HOME ENERGY GRID}

\section{A. Technical Issues}

On the scenario above, there are some technical issues to consider for providing energy management services. Figure 2 illustrates the issues for HEG services in the smart homes, which include three layers. First, hardware layer (HWL) detects instantaneous and accumulated power usage using SPs, which are installed for each appliance. Also, sensors collect and send sensing data, such as occupancy and temperature, to the HEG server. The information, such as name, type and instantaneous power usage, for each appliance is saved in the profiles and used to control the energy saving services. Then, energy-aware layer (EAL) translates the law data to the context information, and decides what the situations are and which services are required. For example, EAL recognizes the energy leakage situation using the information of the occupancy, and power usage. Finally, energy saving services, such as DRLC and RTP, are provided in the application layer (APL), which sends control command to the SPs. Appliances can be turned off because SPs has an ability to shut off from electricity. Residences can control appliances automatic or manual modes. In the automatic mode, an administrative resident defines control rules for DRLC and RTP services in advance, and appliances can be controlled when the services are occurred from the ESPs. In the other mode, appliances are controlled manually by the resident. Also, all residents can monitor and control appliances using their mobile devices according to their permission level.

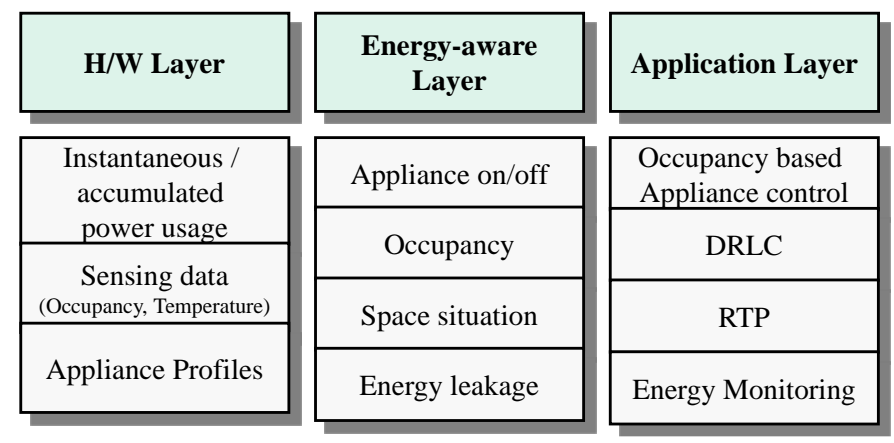

Figure 2. Technical issues for HEG services 


\section{B. System Architecture}

Figure 3 shows the system architecture for HEG services. For the wireless network, Wi-Fi, ZigBee, SUN, and IR are used to communicate among HEG server, SPs, appliances, and sensors. HEG server and SPs are the new system to provide HEG services. The HEG server manages users, devices, sessions, energy information, reports energy-related events to the designated user, and controls appliances to save energy. SPs are collect meter data for each appliance and send them to the HEG server. EAL translates the meter and sensor data into context information, generates energy conservation measures, and controls appliances using the predefined rules. In APL, the energy saving services are provided related to ESPs, which invoke the services, such as DRLC, RTP, energy flow reservation, monitoring, and so on. For example, ESP generates the RTP service, sends the signal to the HEG server. Then, the server detects the mode for the service. There are two types of the modes for the service, such as manual and automatic mode. The designate resident chooses the devices for responding to the service in the manual mode. In the automatic mode, the devices are predefined in advance by the resident before the service is triggered.

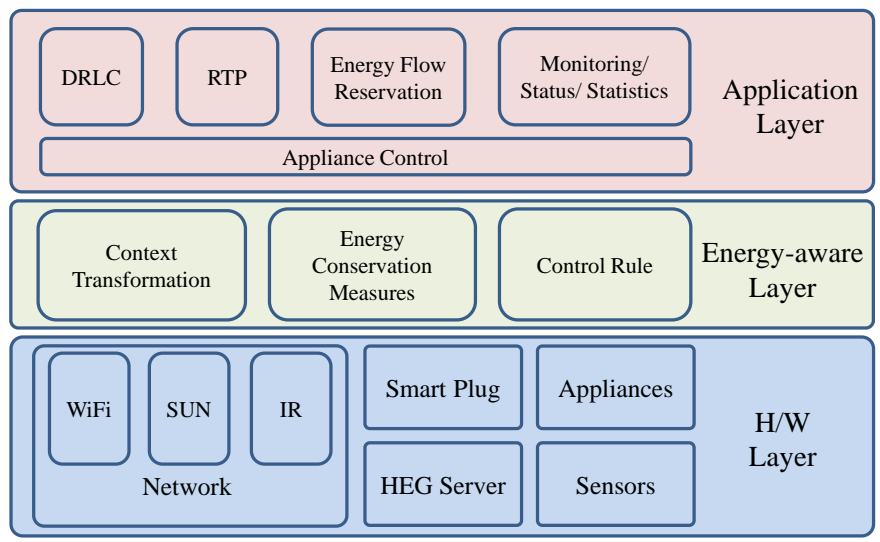

Figure 3. Architecture of HEG system

Figure 4 shows the sequence flow of initial registration of a SP and an appliance. A SP is installed for each appliance. Then it detects the appliance and the HEG server, and sends the registration message to the server. The administrative resident's mobile device operates as the screen of the server, and the informations of the SP and the appliance are input into the server using the mobile device.

The intervals for keepalive message and metering data can also be set. Keepalive messages are for the session among the server and the SPs. Metering data are sent by SPs to the HEG server periodically or on demand by the server requests. Then, the server saves the data for each appliance and for each zone. The residents can see the information using their mobile devices, and control the appliances to reduce energy costs. The messages for turning off appliances are sent from mobile device to the SPs through the server, and they block the power to the appliances. Using this mechanism, users can recognize the energy consumption for their home, and reduce the energy leakage.

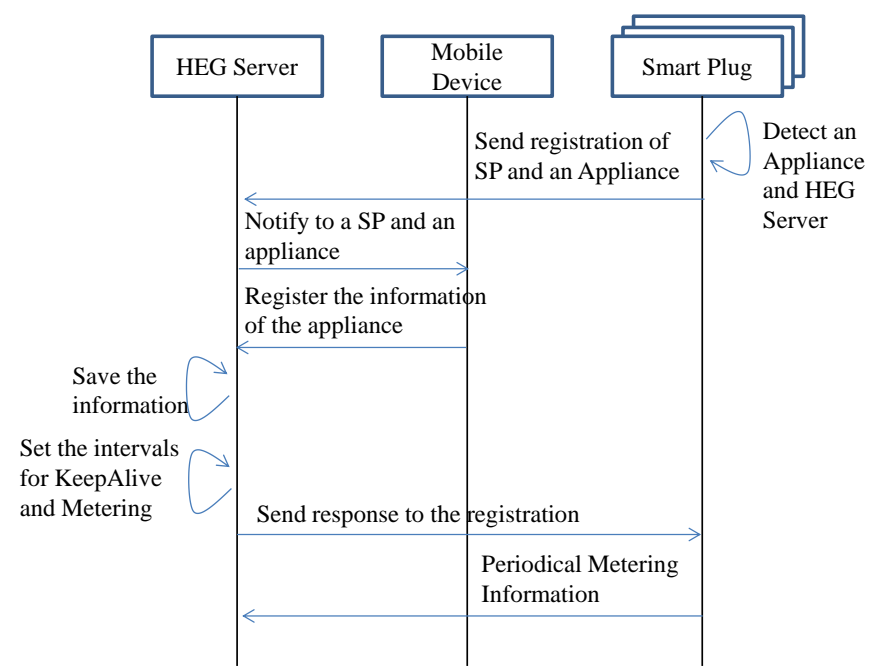

Figure 4. Sequence flow of initial registration of a SP and an appliance

\section{Conclusion}

In this paper, HEG-based smart energy management services were illustrated. SPs were installed for each appliance, and sent the data of power consumption to the HEG server. Then, the server processes, saves, and manages the data to provide energy management services in the smart home. Using the method, users can monitor and control the appliances by their mobile devices through the server and SPs. Also, they can recognize the energy consumption for their home, and reduce the energy leakage by turning off the appliances when not used. As a result, energy costs can be saved by monitoring and controlling the appliances when not in use.

\section{ACKNOWLEDGMENT}

This work was supported in part by the R\&D program of Ministry of Trade, Industry and Energy (MOTIE), Republic of Korea, under Grant of no. 10045739, Development of Energy Management Technology for Home Energy Grid based on Wireless Personal Area Network

\section{REFERENCES}

[1] Hyunjeong Lee, Youn-Kwae Jeong, Il-Woo Lee, Sang Ho Lee, "A Service Framework for Building Energy Management Technology," 2012 International Conference on Convergence Technology, pp.311314, China, July 2012.

[2] Korea Energy Economics Institute, Yearbook of Energy Statistics, vol 31, Dec.2012.

[3] Zhuang Zhao, Won Cheol Lee, Yoan Shin, and Kyung-Bin Song, “An Optimal Power Scheduling Method Applied in Home Energy Management System Based on Demand Response," ETRI Journal, vol 35, no. 4, pp. 677-686, Aug. 2013

[4] H.Y. Kim, J.S. Jeong, W.S. Cha, G.S. Shin, and S.T. Kim, "Technical Trends of AMI and HEMS for Smart Grid Implementation,” 2013 Electronics and Telecommunications Trends, vol. 28, no. 2, pp. 11-19, April 2013.

[5] L. Peretto, "The Role of Measurements in the Smart Grid Era," IEEE Instrum. Meas. Mag., vol. 13, no. 3, June 2010, pp. 22-25. 
[6] ZigBee Alliance and HomePlug Appliance, Smart Energy Profile 2 Application Protocol Standard, April 2013.

[7] Robby Simpson, "Smart Energy Profile 2.0 Overview," ISO/IEC JTC 1/SC 25/WG1, April 2013.

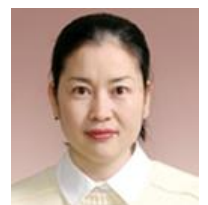

Hyunjeong Lee received her B.S. and M.S. degrees in computer science from Chungbuk National University,

Korea in 1997 and 1999 respectively. She joined the Electronics and Telecommunications Research Institute (ETRI) in 1999. She has been engaged in the research and development of communication protocols, home network service, context-aware framework, content transformation technology and so on. She is currently working as a senior engineer of energy IT technology research section. Her current research interests include multimedia streaming, and energy optimization, and smart grid technology.

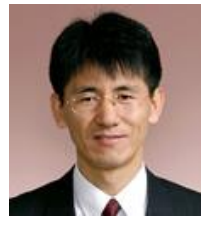

Wan-Ki Park received the B.S. and M.S. degrees in electronics engineering from Chungnam National University, Korea, in 1991 and 1993 respectively and $\mathrm{Ph}$ D. degrees in information Communication Engineering from the same university in 2006. He joined Agency for Defense Development, in 1993 and had been engaged in the research and development of communication related to military till 2000 He joined Electronics and Telecommunications Research Institute, in July 2000 and has been engaged in the research and development of ATM based MPLS system, high speed routing system and home network system, etc. Now, he is principal engineer of energy IT technology research section and his research interests are home network technologies, home digital multimedia services, Green Home/Building and Smart Grid.

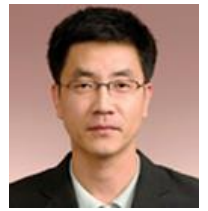

II-Woo Lee received B.S. and M.S. degrees in Computer Engineering from Kyung Hee University, Korea, in 1992 and 1994 respectively and Ph.D degrees in Compute Engineering from Chungnam National University, Korea, in 2007. He joined Electronics and Telecommunications Research Institute (ETRI) in 1994 and has been engaged in the research and development of ATM, CDMA Switching system, home network system and P2P network, etc. Now, he is a head of energy IT technology research section and his research interests are green home/building/industry solutions, smart grid standardization framework, network and energy-IT technology. 